\title{
The Effect of Independent Parameter on Accuracy of Direct Block Method
}

\author{
Iskandar Shah Mohd Zawawi $^{1, *}$, Zarina Bibi Ibrahim ${ }^{2}$, Khairil Iskandar Othman ${ }^{1}$ \\ ${ }^{1}$ Centre of Mathematics Studies, Faculty of Computer and Mathematical Sciences, Kompleks Al-Khawarizmi, Universiti Teknologi \\ MARA Shah Alam, 40450 Shah Alam, Selangor, Malaysia \\ ${ }^{2}$ Department of Mathematics, Faculty of Science, Universiti Putra Malaysia, 43400 Serdang, Selangor, Malaysia
}

Received March 27, 2021; Revised May 6, 2021; Accepted June 15, 2021

\section{Cite This Paper in the following Citation Styles}

(a): [1] Iskandar Shah Mohd Zawawi, Zarina Bibi Ibrahim, Khairil Iskandar Othman, "The Effect of Independent Parameter on Accuracy of Direct Block Method," Mathematics and Statistics, Vol. 9, No. 4, pp. 421 - 430, 2021. DOI: 10.13189/ms.2021.090402.

(b): Iskandar Shah Mohd Zawawi, Zarina Bibi Ibrahim, Khairil Iskandar Othman (2021). The Effect of Independent Parameter on Accuracy of Direct Block Method. Mathematics and Statistics, 9(4), 421 - 430. DOI: $10.13189 / \mathrm{ms} .2021 .090402$.

Copyright $\odot 2021$ by authors, all rights reserved. Authors agree that this article remains permanently open access under the terms of the Creative Commons Attribution License 4.0 International License

\begin{abstract}
Block methods that approximate the solution at several points in block form are commonly used to solve higher order differential equations. Inspired by the literature and ongoing research in this field, this paper intends to explore a new derivation of block backward differentiation formula that employs independent parameter to provide sufficient accuracy when solving second order ordinary differential equations directly. The use of three backward steps and five independent parameters are considered adequately in generating the variable coefficients of the formulas. To ascertain only one parameter exists in the derived formula, the order of the method is determined. Such independent parameter retains the favorable convergence properties although the values of parameter will affect the zero stability and truncation error. An ability of the method to compute the approximated solutions at two points concurrently is undeniable. Another advantage of the method is being able to solve the second order problems directly without recourse to the technique of reducing it to a system of first order equations. The essential of the error analysis is to observe the effect of independent parameter on the accuracy, in the sense that with certain appropriate values of parameter, the accuracy is improved. The performance of the method is tested with some initial value problems and the numerical results confirm that the maximum error and average error obtained by the proposed method are smaller at certain step size compared to the other conventional direct methods.
\end{abstract}

Keywords Block Method, Parameter, Convergence, Ordinary Differential Equations

\section{Introduction}

The mathematical problems in the physical world such as chemical kinetics, vibrations and electrical circuits can be found in the second order ordinary differential equations (ODEs) of the following form:

$$
y^{\prime \prime}=f\left(x, y, y^{\prime}\right),
$$

where $y(a)=y_{0}, y^{\prime}(a)=y_{0}^{\prime}$ are initial conditions with the interval $x \in[a, b]$. Equation (1) can be associated with an equivalent system of first order ODEs as follows:

$$
y_{1}^{\prime}=f\left(x, y_{2}\right), \quad y_{2}^{\prime}=f\left(x, y_{1}, y_{2}\right) .
$$

Equations (2) can be solved numerically using any first order ODEs solver such as backward differentiation formula (BDF) as introduced by [1] and block backward differentiation formulas (BBDF), which was proposed by [2]. However, this approach is cumbersome, consume a lot of human effort and increase the computational time. It will be more efficient if (1) can be solved directly without reducing it to a system of (2). The capability of the numerical methods in solving higher order ODEs directly 
has been studied dramatically by several researchers in the literature. Among the earliest work was proposed by [3] with 3-point explicit-implicit block method for solving special second order ODEs and continuous sixth order explicit method for direct solution of un-damped, linear, nonlinear and system of second order ODEs by [4]. Then, [5] modified the existing block method based on the collocation and interpolation of the power series for the direct solution of second order ODEs. In the recent year, [6] presented a generalized form of the modified Taylor series (MTS) through the modification of the conventional Taylor series approach that produces any $\mathrm{k}$-step block method for solving second order ODEs.

Other famous direct methods were presented by [7] with backward difference formulas for solving higher order ODEs, Runge-Kutta method of order 4 by [8] and fifth order block backward differentiation formulas (5-DBBDF) by [9]. The advantages of direct block method are consistent, convergent, zero stable and ability to approximate the solutions at several points concurrently.

From the literature review, many improvements in numerical methods have been made and it is rationale to say that most of the modified versions tend to affect the accuracy and efficiency of the methods. Our attention here is to propose the direct block backward differentiation formulas (BBDF) with independent parameter which is an improvement on the work of [9]. Contrary to the conventional BBDF method, the proposed method is derived using three backward steps and five independent parameters by adopting the technique presented by [10]-[13] for solving (1) directly without reducing it to (2). This is the idea underlying the derivation of the new version of numerical method, where the value of parameter can be adjusted accordingly to improve the approximation of initial value problems (IVPs). The strategy to derive the method is presented in the following section.

\section{Methodology}

\subsection{Formulation of the Method}

The preliminary step to formulate the method requires the derivation of 2-point block method using constant step size, $h$ where the interval $[a, b]$ is divided into a series of block. The solutions at the previous points, $x_{n-2}, x_{n-1}$ and $x_{n}$ are consumed to compute two numerical solutions, $y_{n+1}$ and $y_{n+2}$ at two points, $x_{n+1}$ and $x_{n+2}$ concurrently. The following equation is the Lagrange interpolation polynomial $P_{k}(x)$ of degree $k=4$ that has been used as the basis functions:

$$
P_{k}(x)=\sum_{j=0}^{k} L_{k, j}(x) y\left(x_{n+2-j}\right)
$$

where

$$
L_{k, j}(x)=\prod_{\substack{i=0 \\ i \neq j}}^{k} \frac{\left(x-x_{n+2-i}\right)}{\left(x_{n+2-j}-x_{n+2-i}\right)}
$$

for each $j=0,1, \ldots, k$ and $n$ is the grid index.

Define $x=x_{n+1}+s h$ to find the interpolating polynomial of degree 4:

$$
\begin{aligned}
P_{4}\left(x_{n+1}+s h\right) & =\left(\frac{-2 s-s^{2}+2 s^{3}+s^{4}}{24}\right) y_{n-2}+\left(\frac{3 s+s^{2}-3 s^{3}-s^{4}}{6}\right) y_{n-1}+\left(\frac{-6 s+s^{2}+4 s^{3}+s^{4}}{4}\right) y_{n} \\
& +\left(\frac{1+5 s-5 s^{2}-5 s^{3}-s^{4}}{6}\right) y_{n+1}+\left(\frac{6 s+11 s^{2}+6 s^{3}+s^{4}}{24}\right) y_{n+2} .
\end{aligned}
$$

Consequently, (4) is differentiated twice with respect to $s$ at the point $x=x_{n+1}$ to produce

$$
\begin{aligned}
P_{4} "\left(x_{n+1}+s h\right) & =\left(\frac{-2+12 s+12 s^{2}}{24}\right) y_{n-2}+\left(\frac{2-18 s-12 s^{2}}{6}\right) y_{n-1}+\left(\frac{2+24 s+12 s^{2}}{4}\right) y_{n} \\
& +\left(\frac{-10-30 s-12 s^{2}}{6}\right) y_{n+1}+\left(\frac{22+36 s+12 s^{2}}{24}\right) y_{n+2} .
\end{aligned}
$$

Let $s=0$, gives

$$
\begin{aligned}
& P^{\prime}\left(x_{n+1}\right)=h y_{n+1}^{\prime}=-\frac{1}{12} y_{n-2}+\frac{1}{2} y_{n-1}-\frac{3}{2} y_{n}+\frac{5}{6} y_{n+1}+\frac{1}{4} y_{n+2}, \\
& P^{\prime \prime}\left(x_{n+1}\right)=h^{2} y_{n+1}^{\prime \prime}=-\frac{1}{12} y_{n-2}+\frac{1}{3} y_{n-1}+\frac{1}{2} y_{n}-\frac{5}{3} y_{n+1}+\frac{11}{12} y_{n+2} .
\end{aligned}
$$


Let $s=1$, produces

$$
\begin{aligned}
& P^{\prime}\left(x_{n+2}\right)=h y_{n+2}^{\prime}=\frac{1}{4} y_{n-2}-\frac{4}{3} y_{n-1}+3 y_{n}-4 y_{n+1}+\frac{25}{12} y_{n+2}, \\
& P^{\prime \prime}\left(x_{n+2}\right)=h^{2} y_{n+2}^{\prime \prime}=\frac{11}{12} y_{n-2}-\frac{14}{3} y_{n-1}+\frac{19}{2} y_{n}-\frac{26}{3} y_{n+1}+\frac{35}{12} y_{n+2} .
\end{aligned}
$$

By considering $y_{n+1}^{\prime \prime}=f_{n+1}$ and $y_{n+2}^{\prime \prime}=f_{n+2}$, the following equations are obtained:

$$
\begin{aligned}
y_{n+1}^{\prime} & =\frac{1}{h}\left(-\frac{1}{12} y_{n-2}+\frac{1}{2} y_{n-1}-\frac{3}{2} y_{n}+\frac{5}{6} y_{n+1}+\frac{1}{4} y_{n+2}\right), \\
y_{n+1} & =-\frac{1}{20} y_{n-2}+\frac{1}{5} y_{n-1}+\frac{3}{10} y_{n}+\frac{11}{20} y_{n+2}-\frac{3}{5} h^{2} f_{n+1}, \\
y_{n+2}^{\prime} & =\frac{1}{h}\left(\frac{1}{4} y_{n-2}-\frac{4}{3} y_{n-1}+3 y_{n}-4 y_{n+1}+\frac{25}{12} y_{n+2}\right), \\
y_{n+2} & =-\frac{11}{35} y_{n-2}+\frac{8}{5} y_{n-1}-\frac{114}{35} y_{n}+\frac{104}{35} y_{n+1}+\frac{12}{35} h^{2} f_{n+2} .
\end{aligned}
$$

While the derivation of block method is rather straightforward, the same is not true for the block method with independent parameter. Although (8) has been explored by [9], none this approach presented a formula with independent parameter. The strategy to add parameters in (8) is similar to the one used for BDF with three parameters, $\alpha, \beta$ and $\gamma$ as presented in [10]. Therefore, we consider 5 parameters $\alpha, \beta, \rho, \mu$ and $\delta$ for a modification of (8) which can be expressed as follows:

$$
\begin{aligned}
& -\frac{1}{12} y_{n-2}+\frac{1}{2}\left((1+\delta) y_{n-1}-\delta y_{n-2}\right)-\frac{3}{2}\left((1+\mu) y_{n}-\mu y_{n-1}\right)+\frac{5}{6}\left((1+\rho) y_{n+1}-\rho y_{n}\right)+\frac{1}{4}\left((1+\beta) y_{n+2}-\beta y_{n+1}\right) \\
& =h\left((1+\alpha) y_{n+1}^{\prime}-\alpha y_{n}^{\prime}\right), \\
& -\frac{1}{12} y_{n-2}+\frac{1}{3}\left((1+\delta) y_{n-1}-\delta y_{n-2}\right)+\frac{1}{2}\left((1+\mu) y_{n}-\mu y_{n-1}\right)-\frac{5}{3}\left((1+\rho) y_{n+1}-\rho y_{n}\right)+\frac{11}{12}\left((1+\beta) y_{n+2}-\beta y_{n+1}\right) \\
& =h^{2}\left((1+\alpha) f_{n+1}-\alpha f_{n}\right), \\
& \frac{1}{4} y_{n-2}-\frac{4}{3}\left((1+\delta) y_{n-1}-\delta y_{n-2}\right)+3\left((1+\mu) y_{n}-\mu y_{n-1}\right)-4\left((1+\rho) y_{n+1}-\rho y_{n}\right)+\frac{25}{12}\left((1+\beta) y_{n+2}-\beta y_{n+1}\right) \\
& =h\left((1+\alpha) y_{n+2}^{\prime}-\alpha y_{n+1}^{\prime}\right), \\
& \frac{11}{12} y_{n-2}-\frac{14}{3}\left((1+\delta) y_{n-1}-\delta y_{n-2}\right)+\frac{19}{2}\left((1+\mu) y_{n}-\mu y_{n-1}\right)-\frac{26}{3}\left((1+\rho) y_{n+1}-\rho y_{n}\right)+\frac{35}{12}\left((1+\beta) y_{n+2}-\beta y_{n+1}\right) \\
& =h^{2}\left((1+\alpha) f_{n+2}-\alpha f_{n+1}\right) .
\end{aligned}
$$

Meanwhile, additional effort is required in transforming (9) into the formula that has only one independent parameter $\alpha$. This will be discussed in the next section.

\subsection{Order of the Method}

Formulas (9) correspond to the standard linear multistep method (LMM) in the following form:

$$
\sum_{j=0}^{k} A_{j} y_{n+j-2}=h \sum_{j=0}^{k} B_{j} y_{n+j-2}^{\prime}+h^{2} \sum_{j=0}^{k} D_{j} y_{n+j-2}^{\prime \prime},
$$

where $A_{j}, B_{j}$ and $D_{j}$ are constant of matrix coefficients subject to the conditions $A_{k} \neq 0$, where $k=4$ and not all $A_{0}, B_{0}$ and $D_{0}$ are zero. With the LMM (10), the associated linear difference operator, $L$ is defined as

$$
L[y(x) ; h]=\sum_{j=0}^{k}\left[A_{j} y(x+j h)-h B_{j} y^{\prime}(x+j h)-h^{2} D_{j} y^{\prime \prime}(x+j h)\right],
$$

where $y(x)$ be any twice differentiable function on $[a, b]$, which may have as many higher derivatives. The order of 
accuracy of (10) and (11) can be defined formally without invoking the solution of (1) which may possess only a first derivative [14]. By using the Taylor series to expand the functions $y(x+j h), y^{\prime}(x+j h)$ and $y^{\prime \prime}(x+j h)$ about $x+j h$ and collecting terms in (11), gives

$$
L[y(x) ; h]=C_{0} y(x)+C_{1} h y^{\prime}(x)+C_{2} h^{2} y^{\prime \prime}(x)+\cdots+C_{q} h^{q} y^{(q)}(x) .
$$

Definition: The LMM (10) and linear operator (11) are said to be of order $q$ if $C_{0}=C_{1}=\ldots=C_{q}=C_{q+1}=0, C_{q+2} \neq 0$ where $C_{q+2}$ is error constant. The constants $C_{0}, C_{1}$ and $C_{q}$ are defined as

$$
C_{0}=\sum_{j=0}^{k} A_{j}, C_{1}=\sum_{j=0}^{k}\left(j A_{j}-B_{j}\right), C_{q}=\sum_{j=0}^{k}\left(j^{q} \frac{1}{q !} A_{j}-j^{q-1} \frac{1}{(q-1) !} B_{j}-j^{q-2} \frac{1}{(q-2) !} D_{j}\right), q=2,3,4, \cdots .
$$

To determine the order of the method, the matrix coefficients of (9) which corresponds to (10) can be written as follows:

$$
\begin{gathered}
A_{0}=\left[\begin{array}{c}
-\frac{1}{12}-\frac{1}{2} \delta \\
-\frac{1}{12}-\frac{1}{3} \delta \\
\frac{1}{4}+\frac{4}{3} \delta \\
\frac{11}{12}+\frac{14}{3} \delta
\end{array}\right], A_{1}=\left[\begin{array}{c}
\frac{1}{2}+\frac{1}{2} \delta+\frac{3}{2} \mu \\
\frac{1}{3}+\frac{1}{3} \delta-\frac{1}{2} \mu \\
-\frac{4}{3}-3 \mu-\frac{4}{3} \delta \\
-\frac{19}{2} \mu-\frac{14}{3}-\frac{14}{3} \delta
\end{array}\right], A_{2}=\left[\begin{array}{c}
-\frac{3}{2}-\frac{3}{2} \mu-\frac{5}{6} \rho \\
\frac{1}{2}+\frac{1}{2} \mu+\frac{5}{3} \rho \\
3+4 \rho+3 \mu \\
\frac{19}{2}+\frac{19}{2} \mu+\frac{26}{3} \rho
\end{array}\right], A_{3}=\left[\begin{array}{c}
\frac{5}{6}+\frac{5}{6} \rho-\frac{1}{4} \beta \\
-\frac{5}{3}-\frac{5}{3} \rho-\frac{11}{12} \beta \\
-4-4 \rho-\frac{25}{12} \beta \\
-\frac{26}{3}-\frac{35}{12} \beta-\frac{26}{3} \rho
\end{array}\right], A_{4}=\left[\begin{array}{c}
\frac{1}{4}+\frac{1}{4} \beta \\
\frac{11}{12}+\frac{11}{12} \beta \\
\frac{25}{12}+\frac{25}{12} \beta \\
\frac{35}{12}+\frac{35}{12} \beta
\end{array}\right], \\
B_{2}=\left[\begin{array}{c}
-\alpha \\
0 \\
0 \\
0
\end{array}\right], B_{3}=\left[\begin{array}{c}
1+\alpha \\
0 \\
-\alpha \\
0
\end{array}\right], B_{4}=\left[\begin{array}{c}
0 \\
0 \\
1+\alpha \\
0
\end{array}\right], D_{2}=\left[\begin{array}{c}
0 \\
-\alpha \\
0 \\
0
\end{array}\right], D_{3}=\left[\begin{array}{c}
0 \\
1+\alpha \\
0 \\
-\alpha
\end{array}\right], D_{4}=\left[\begin{array}{c}
0 \\
0 \\
0 \\
1+\alpha
\end{array}\right] .
\end{gathered}
$$

Therefore, the values of $C_{q}=\left[\begin{array}{llll}C_{q, 1} & C_{q, 2} & C_{q, 3} & C_{q, 4}\end{array}\right]^{T}, q=0, \ldots, 5$ are obtained below:

$$
\begin{gathered}
C_{0}=\sum_{j=0}^{4} A_{j}=\left[\begin{array}{l}
0 \\
0 \\
0 \\
0
\end{array}\right], \\
C_{1}=\sum_{j=0}^{4}\left(j A_{j}-B_{j}\right)=\left[\begin{array}{c}
\frac{1}{2} \delta-\frac{3}{2} \mu+\frac{5}{6} \rho+\frac{1}{4} \beta \\
\frac{1}{3} \delta+\frac{1}{2} \mu-\frac{5}{3} \rho+\frac{11}{12} \beta \\
-\frac{4}{3} \delta+3 \mu-4 \rho+\frac{25}{12} \beta \\
-\frac{14}{3} \delta+\frac{19}{2} \mu-\frac{26}{3} \rho+\frac{35}{12} \beta
\end{array}\right], \\
C_{2}=\sum_{j=0}^{4}\left(\frac{1}{2} j^{2} A_{j}-j B_{j}-D_{j}\right)=\left[\begin{array}{c}
\frac{1}{4} \delta-\frac{9}{4} \mu+\frac{25}{12} \rho+\frac{7}{8} \beta-\alpha \\
\frac{1}{6} \delta+\frac{3}{4} \mu-\frac{25}{6} \rho+\frac{77}{24} \beta \\
-\frac{2}{3} \delta+\frac{9}{2} \mu-10 \rho+\frac{175}{24} \beta-\alpha \\
-\frac{7}{3} \delta+\frac{57}{4} \mu-\frac{65}{3} \rho+\frac{245}{24} \beta
\end{array}\right],
\end{gathered}
$$




$$
\begin{gathered}
C_{4}=\sum_{j=0}^{4}\left(\frac{1}{24} j^{4} A_{j}-\frac{1}{6} j^{3} B_{j}-\frac{1}{2} j^{2} D_{j}\right)=\left[\begin{array}{c}
\frac{1}{12} \delta-\frac{7}{4} \mu+\frac{95}{36} \rho+\frac{37}{24} \beta-\frac{5}{2} \alpha \\
\frac{1}{18} \delta+\frac{7}{12} \mu-\frac{95}{18} \rho+\frac{407}{72} \beta-\alpha \\
-\frac{2}{9} \delta+\frac{7}{2} \mu-\frac{38}{3} \rho+\frac{925}{72} \beta-\frac{7}{2} \alpha \\
-\frac{7}{9} \delta+\frac{133}{12} \mu-\frac{247}{9} \rho+\frac{1295}{72} \beta-\alpha
\end{array}\right], \\
C_{5}=\sum_{j=0}^{4}\left(\frac{1}{120} j^{5} A_{j}-\frac{1}{24} j^{4} B_{j}-\frac{1}{6} j^{3} D_{j}\right)=\left[\begin{array}{c}
\frac{1}{20}+\frac{1}{240} \delta-\frac{31}{80} \mu+\frac{211}{144} \rho+\frac{781}{480} \beta-\frac{65}{24} \alpha \\
\frac{1}{12}+\frac{1}{360} \delta+\frac{31}{240} \mu-\frac{211}{72} \rho+\frac{8591}{1440} \beta-\frac{19}{6} \alpha \\
-\frac{1}{5}-\frac{1}{90} \delta+\frac{31}{40} \mu-\frac{211}{30} \rho+\frac{3905}{288} \beta-\frac{175}{24} \alpha \\
-\frac{5}{6}-\frac{7}{180} \delta+\frac{589}{240} \mu-\frac{2743}{180} \rho+\frac{5467}{288} \beta-\frac{37}{6} \alpha
\end{array}\right] .
\end{gathered}
$$

By solving $C_{q}, q=0, \ldots, 4$ simultaneously, the conditions to verify the order of the method are obtained as follows:

$$
\begin{aligned}
& \mu=\frac{2}{3} \alpha, \beta=\frac{4}{3} \alpha, \rho=\frac{3}{5} \alpha, \delta=\frac{1}{3} \alpha, \\
& \mu=2 \alpha, \beta=\frac{12}{11} \alpha, \rho=\frac{6}{5} \alpha, \delta=0, \\
& \mu=\frac{1}{2} \alpha, \beta=\frac{22}{25} \alpha, \rho=\frac{3}{4} \alpha, \delta=\frac{1}{4} \alpha, \\
& \mu=\frac{8}{19} \alpha, \beta=\frac{24}{35} \alpha, \rho=\frac{15}{26} \alpha, \delta=\frac{3}{14} \alpha,
\end{aligned}
$$

The conditions (13) - (16) are substituted into $C_{q, 1}, C_{q, 2}, C_{q, 3}$ and $C_{q, 4}$ respectively to obtain

$$
C_{1}=C_{2}=C_{3}=C_{4}=\left[\begin{array}{llll}
0 & 0 & 0 & 0
\end{array}\right]^{T} \text { and } C_{5}=\left[\frac{1}{20}+\frac{1}{12} \alpha \frac{1}{12}+\frac{1}{12} \alpha-\frac{1}{20} \alpha-\frac{31}{30}-\frac{67}{60} \alpha\right]^{T} \text {, }
$$

where $C_{5}$ is the error constant. Since $C_{1}=C_{2}=C_{3}=C_{4}=0$ and $C_{5} \neq 0$, it can be concluded that the derived formula is order 3. By substituting the conditions (13) - (16) into (9), the desired formula that has one independent parameter $\alpha$ is obtained as follows:

$$
\begin{gathered}
(1+\alpha) h y_{n+1}^{\prime}=\left(\frac{5}{6}+\frac{1}{6} \alpha\right) y_{n+1}+\left(\frac{1}{4}+\frac{1}{3} \alpha\right) y_{n+2}+\left(-\frac{3}{2}-\frac{3}{2} \alpha\right) y_{n}+\left(\frac{1}{2}+\frac{7}{6} \alpha\right) y_{n-1}+\left(-\frac{1}{12}-\frac{1}{6} \alpha\right) y_{n-2}+\alpha h y_{n}^{\prime} \\
(1+\alpha) h y_{n+2}^{\prime}=\left(-4-\frac{29}{6} \alpha\right) y_{n+1}+\left(\frac{25}{12}+\frac{11}{6} \alpha\right) y_{n+2}+\left(3+\frac{9}{2} \alpha\right) y_{n}+\left(-\frac{4}{3}-\frac{11}{6} \alpha\right) y_{n-1}+\left(\frac{1}{4}+\frac{1}{3} \alpha\right) y_{n-2}+\alpha h y_{n+1}^{\prime} \\
\left(-\frac{5}{3}-3 \alpha\right) y_{n+1}=\left(-\frac{11}{12}-\alpha\right) y_{n+2}+\left(-\frac{1}{2}-3 \alpha\right) y_{n}+\left(-\frac{1}{3}+\alpha\right) y_{n-1}+\frac{1}{12} y_{n-2}+(1+\alpha) h^{2} f_{n+1}-\alpha h^{2} f_{n} \\
\left(\frac{35}{12}+2 \alpha\right) y_{n+2}=\left(\frac{26}{3}+7 \alpha\right) y_{n+1}+\left(-\frac{19}{2}-9 \alpha\right) y_{n}+\left(5 \alpha+\frac{14}{3}\right) y_{n-1}+\left(-\frac{11}{12}-\alpha\right) y_{n-2}+(1+\alpha) h^{2} f_{n+2}-\alpha h^{2} f_{n+1}
\end{gathered}
$$

Since $\alpha$ is the only parameter that represents all parameters, this formula (17) is called BBDF2- $\alpha$. It has to be noted that the existence of $\alpha$ in the error constant, $C_{5}$ will affect the magnitude of the truncation error. This means the error might grow if we increase the value of $\alpha$. Therefore, it is advisable to choose an appropriate value of parameter $\alpha$. In this study, we choose $\alpha=-0.3,0.3$ to implement the method. 


\subsection{Convergence Properties}

A practical criterion for the method to be useful is that it satisfies the convergence properties. In this section, the convergence is verified by showing the consistency and zero stability of the BBDF2- $\alpha$.

\subsubsection{Consistency}

Formula (17) is written in the form of (10) to produce the following matrix coefficients:

$$
\begin{gathered}
A_{0}=\left[\begin{array}{c}
-\frac{1}{12}-\frac{1}{6} \alpha \\
-\frac{1}{12} \\
\frac{1}{4}+\frac{1}{3} \alpha \\
\frac{11}{12}+\alpha
\end{array}\right], A_{1}=\left[\begin{array}{c}
\frac{1}{2}+\frac{7}{6} \alpha \\
\frac{1}{3}-\alpha \\
-\frac{4}{3}-\frac{11}{6} \alpha \\
-5 \alpha-\frac{14}{3}
\end{array}\right], A_{2}=\left[\begin{array}{c}
-\frac{3}{2}-\frac{3}{2} \alpha \\
\frac{1}{2}+3 \alpha \\
3+\frac{9}{2} \alpha \\
\frac{19}{2}+9 \alpha
\end{array}\right], A_{3}=\left[\begin{array}{c}
\frac{5}{6}+\frac{1}{6} \alpha \\
-\frac{5}{3}-3 \alpha \\
-4-\frac{29}{6} \alpha \\
-\frac{26}{3}-7 \alpha
\end{array}\right], A_{4}=\left[\begin{array}{c}
\frac{1}{4}+\frac{1}{3} \alpha \\
\frac{11}{12}+\alpha \\
\frac{25}{12}+\frac{11}{6} \alpha \\
\frac{35}{12}+2 \alpha
\end{array}\right], \\
B_{2}=\left[\begin{array}{c}
-\alpha \\
0 \\
0 \\
0
\end{array}\right], B_{3}=\left[\begin{array}{c}
1+\alpha \\
0 \\
-\alpha \\
0
\end{array}\right], B_{4}=\left[\begin{array}{c}
0 \\
0 \\
1+\alpha \\
0
\end{array}\right], D_{2}=\left[\begin{array}{c}
0 \\
-\alpha \\
0 \\
0
\end{array}\right], D_{3}=\left[\begin{array}{c}
0 \\
1+\alpha \\
0 \\
-\alpha
\end{array}\right], D_{4}=\left[\begin{array}{c}
0 \\
0 \\
0 \\
1+\alpha
\end{array}\right] .
\end{gathered}
$$

Formula (17) is said to be consistent if the following conditions are satisfied:

$$
\begin{gathered}
\sum_{j=0}^{4} A_{j}=A_{0}+A_{1}+A_{2}+A_{3}+A_{4}=\left[\begin{array}{l}
0 \\
0 \\
0 \\
0
\end{array}\right], \sum_{j=0}^{4} j A_{j}=0\left(A_{0}\right)+1\left(A_{1}\right)+2\left(A_{2}\right)+3\left(A_{3}\right)+4\left(A_{4}\right)=\left[\begin{array}{l}
1 \\
0 \\
1 \\
0
\end{array}\right], \\
\sum_{j=0}^{4} B_{j}=B_{0}+B_{1}+B_{2}+B_{3}+B_{4}=\left[\begin{array}{l}
1 \\
0 \\
1 \\
0
\end{array}\right] .
\end{gathered}
$$

Since $\sum_{j=0}^{4} A_{j}=0$ and $\sum_{j=0}^{4} j A_{j}=\sum_{j=0}^{4} B_{j}$, the BBDF2- $\alpha$ is consistent.

\subsubsection{Zero Stability}

The zero stability of the BBDF2- $\alpha$ is investigated to meet the requirement of convergence properties. The stability polynomial associated with formula (17) is accomplished by invoking the standard linear test equation:

$$
f=y^{\prime \prime}=\theta y^{\prime}+\mu y \text {. }
$$

Firstly, we substitute (18) into (17). Then, the equations are written in the matrix form. By assuming $H 1=h^{2} \mu$ and $H 2=h \theta$, the stability polynomial, $p(t)$ associated with BBDF2- $\alpha$ is given by $p(t)=\left|A t^{2}-B t-C\right|=0$. To determine the zero stability of the method, let $H 1=H 2=0$ and gives

$$
\begin{aligned}
p(t)= & -\frac{t^{8}\left(222+660 \alpha+72 \alpha^{4}+726 \alpha^{2}+360 \alpha^{3}\right)}{2(1+\alpha)^{2}(5+9 \alpha)(35+24 \alpha)}-\frac{t^{7}\left(-450-1404 \alpha-288 \alpha^{4}-1896 \alpha^{2}-1152 \alpha^{3}\right)}{2(1+\alpha)^{2}(5+9 \alpha)(35+24 \alpha)} \\
& -\frac{t^{6}\left(234+828 \alpha+432 \alpha^{4}+1620 \alpha^{2}+1296 \alpha^{3}\right)}{2(1+\alpha)^{2}(5+9 \alpha)(35+24 \alpha)}-\frac{t^{5}\left(-6-84 \alpha-456 \alpha^{2}-288 \alpha^{4}-576 \alpha^{3}\right)}{2(1+\alpha)^{2}(5+9 \alpha)(35+24 \alpha)} \\
& -\frac{t^{4}\left(72 \alpha^{4}+6 \alpha^{2}+72 \alpha^{3}\right)}{2(1+\alpha)^{2}(5+9 \alpha)(35+24 \alpha)} .
\end{aligned}
$$

Solve (19) to obtain the roots of stability, $t$ as follows: 


$$
t_{1}=t_{2}=t_{3}=t_{4}=0, t_{5}=t_{6}=1, t_{7}=\frac{\alpha^{2}}{\alpha^{2}+2 \alpha+1}, t_{8}=\frac{12 \alpha^{2}+12 \alpha+1}{12 \alpha^{2}+36 \alpha+37} .
$$

Since the roots of zero stability, $t_{7}$ and $t_{8}$ possess $\alpha$, the graphs of $t_{7}$ and $t_{8}$ versus $\alpha$ are illustrated in Figure 1 . Graph of $t_{7}$ versus and Figure 2 respectively. In figure 1, Graph of $t_{7}$ versus, it can be observed that the values of $t_{7}$ is less than 1 when $\alpha \in[-0.46, \infty)$. Figure 2 shows that $t_{8}<1$ when $\alpha \in[-1.43, \infty)$. Therefore, it can be concluded that the method is zero-stable by considering the values of $\alpha$ in the range $\alpha \in[-0.46, \infty)$.

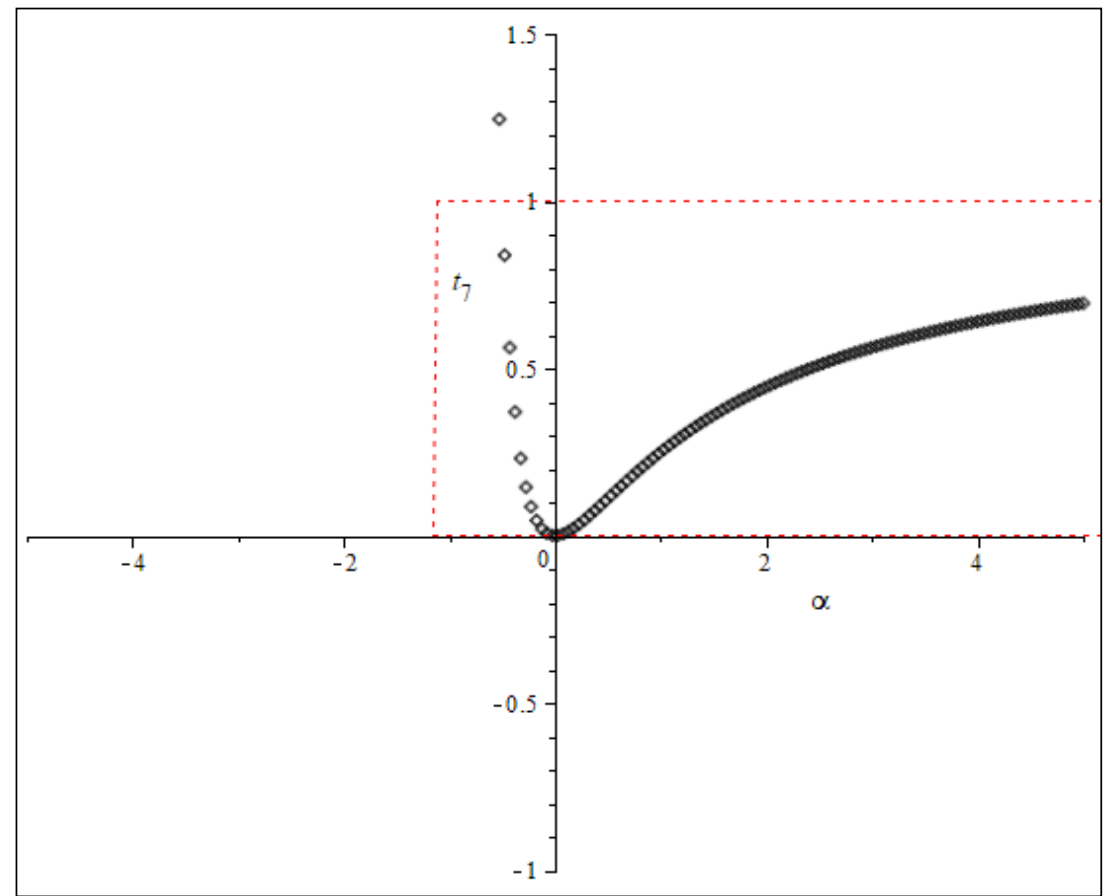

Figure 1. Graph of $t_{7}$ versus $\alpha$

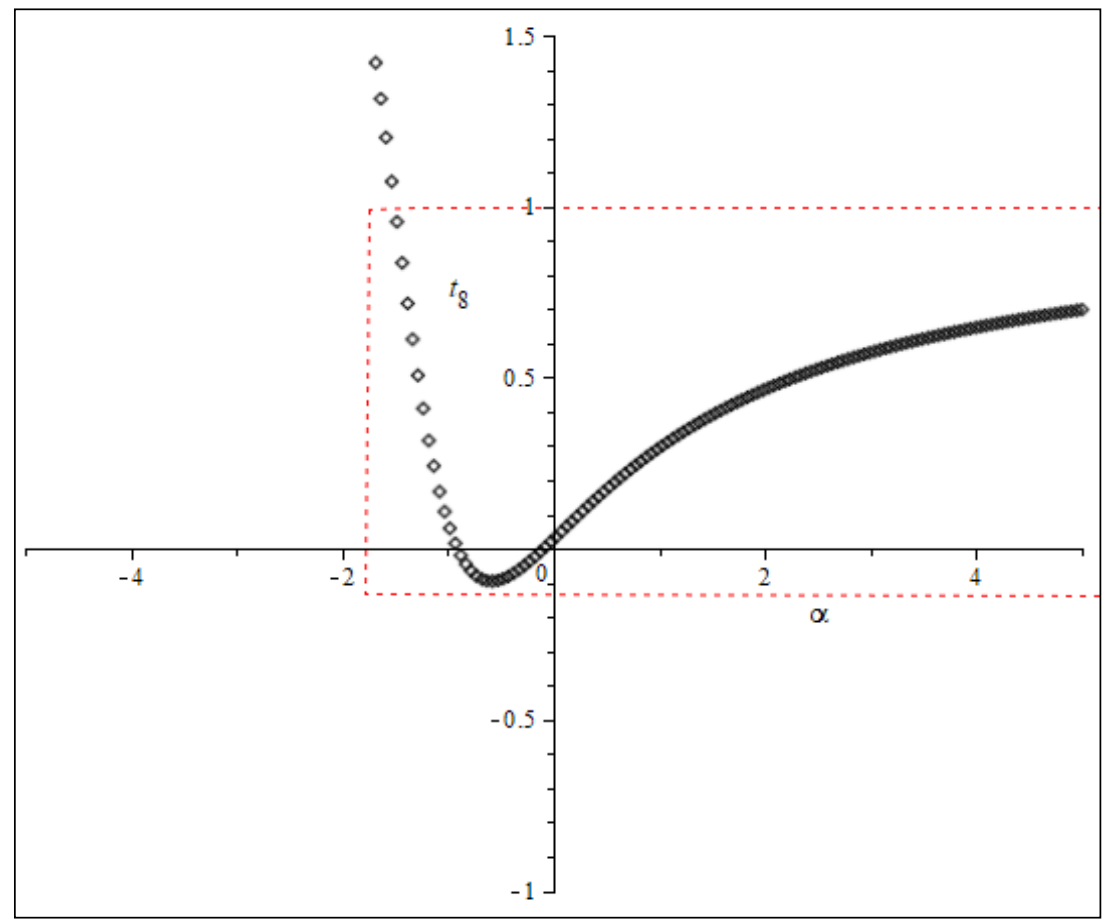

Figure 2. Graph of $t_{8}$ versus $\alpha$ 


\section{Numerical Results}

The C programming language is developed to implement the derived method and Newton's iteration procedures. A significant advantage of using Newton's iteration is the ease of estimation of error. To test the performance of the method, two IVPs of second order ODEs taken from [9] are considered. For each step size, $h$ the performance of the BBDF2- $\alpha$, third order backward differentiation formulas (BDF) [1], fifth order of Gear's method (5-GEAR) [15], third order block backward differentiation formulas (BBDF) [16], third order direct block backward differentiation formulas (DBBDF) [17] and fifth order direct block backward differentiation formulas (5-DBBDF) [9] are compared in terms of total number of steps (TS), maximum error (MAXE) and average error (AVER).

Problem 1. Consider the IVPs of second order linear ODEs as follows:

$$
y^{\prime \prime}(x)=-4000 y(x)-40 y^{\prime}(x)+24,
$$

subject to initial condition

$$
y(0)=0, y^{\prime}(0)=0,0 \leq x \leq 2,
$$

whose exact solution:

$$
y(x)=e^{-20 x}\left(-\frac{3}{500} \cos 60 x-\frac{1}{500} \sin 60 x\right)+\frac{3}{500} .
$$

The following equations are the system of first order ODEs obtained by reducing the order of Problem 1:

$$
\begin{gathered}
y_{1}{ }^{\prime}(x)=y_{2}(x), y_{2}{ }^{\prime}(x)=-4000 y_{1}(x)-40 y_{2}(x)+24, \\
y_{1}(0)=0, y_{2}(0)=0,0 \leq x \leq 2,
\end{gathered}
$$

where the exact solutions are

$$
\begin{aligned}
y_{1}(x)= & e^{-20 x}\left(-\frac{3}{500} \cos 60 x-\frac{1}{500} \sin 60 x\right)+\frac{3}{500}, \\
y_{2}(x) & =-20 e^{-20 x}\left(-\frac{3}{500} \cos 60 x-\frac{1}{500} \sin 60 x\right) \\
& +e^{-20 x}\left(-\frac{3}{25} \cos 60 x+\frac{9}{25} \sin 60 x\right) .
\end{aligned}
$$

Problem 2. The IVPs of second order linear ODEs is given as follows:

$$
y^{\prime \prime}(x)=-5000 y(x)-125 y^{\prime}(x),
$$

\begin{tabular}{|c|c|c|c|c|}
\hline$h$ & Methods & TS & MAXE & AVER \\
\hline \multirow{7}{*}{$10^{-2}$} & $\mathrm{BDF}$ & - & - & - \\
\hline & 5-GEAR & $2 \mathrm{E} 2$ & $2.1696 \mathrm{E}-01$ & $5.1580 \mathrm{E}-03$ \\
\hline & $\mathrm{BBDF}$ & - & - & - \\
\hline & 5-DBBDF & $1 \mathrm{E} 2$ & $1.0382 \mathrm{E}-03$ & $2.5526 \mathrm{E}-05$ \\
\hline & DBBDF & $1 \mathrm{E} 2$ & $1.5630 \mathrm{E}-03$ & $2.8464 \mathrm{E}-05$ \\
\hline & BBDF2- $\alpha=-0.3$ & $1 \mathrm{E} 2$ & $1.5286 \mathrm{E}-03$ & $3.9967 \mathrm{E}-05$ \\
\hline & BBDF2- $\alpha=0.3$ & $1 \mathrm{E} 2$ & $1.5814 \mathrm{E}-03$ & $2.9852 \mathrm{E}-05$ \\
\hline \multirow{7}{*}{$10^{-4}$} & $\mathrm{BDF}$ & $2 \mathrm{E} 4$ & $2.8332 \mathrm{E}-03$ & $1.3236 \mathrm{E}-04$ \\
\hline & 5-GEAR & $2 \mathrm{E} 4$ & $3.5666 \mathrm{E}-05$ & $9.1579 \mathrm{E}-07$ \\
\hline & $\mathrm{BBDF}$ & $1 \mathrm{E} 4$ & $5.7274 \mathrm{E}-03$ & $2.6884 \mathrm{E}-04$ \\
\hline & 5-DBBDF & $1 \mathrm{E} 4$ & 2.9821E-07 & 7.4301E-09 \\
\hline & DBBDF & $1 \mathrm{E} 4$ & $1.8758 \mathrm{E}-07$ & 4.6691E-09 \\
\hline & BBDF2- $\alpha=-0.3$ & $1 \mathrm{E} 4$ & $1.7788 \mathrm{E}-07$ & 4.4463E-09 \\
\hline & BBDF2- $\alpha=0.3$ & $1 \mathrm{E} 4$ & $1.9067 \mathrm{E}-07$ & 4.5187E-09 \\
\hline \multirow{7}{*}{$10^{-6}$} & $\mathrm{BDF}$ & 2E6 & $2.8056 \mathrm{E}-05$ & $1.3054 \mathrm{E}-06$ \\
\hline & 5-GEAR & 2E6 & $3.5719 \mathrm{E}-09$ & $9.2432 \mathrm{E}-11$ \\
\hline & BBDF & 1E6 & $5.6118 \mathrm{E}-05$ & $2.6112 \mathrm{E}-06$ \\
\hline & 5-DBBDF & 1E6 & $6.4142 \mathrm{E}-11$ & $3.0288 \mathrm{E}-11$ \\
\hline & DBBDF & 1E6 & $4.3004 \mathrm{E}-10$ & $1.7870 \mathrm{E}-10$ \\
\hline & BBDF2- $\alpha=-0.3$ & 1E6 & $8.9451 \mathrm{E}-11$ & $6.3772 \mathrm{E}-11$ \\
\hline & BBDF2- $\alpha=0.3$ & 1E6 & $8.0416 \mathrm{E}-10$ & $6.0031 \mathrm{E}-10$ \\
\hline
\end{tabular}

subject to initial condition

$$
y(0)=0, y^{\prime}(0)=4,0 \leq x \leq 2,
$$

\begin{tabular}{|c|c|c|c|c|}
\hline$h$ & Methods & TS & MAXE & AVER \\
\hline \multirow[t]{7}{*}{$10^{-2}$} & $\mathrm{BDF}$ & - & $7.8112 \mathrm{E}-01$ & $1.4632 \mathrm{E}-02$ \\
\hline & 5-GEAR & $2 \mathrm{E} 2$ & $2.5638 \mathrm{E}-01$ & $1.0332 \mathrm{E}-02$ \\
\hline & BBDF & - & $2.0846 \mathrm{E}+03$ & $1.8448 \mathrm{E}+02$ \\
\hline & 5-DBBDF & $1 \mathrm{E} 2$ & $8.8759 \mathrm{E}-04$ & $7.1286 \mathrm{E}-06$ \\
\hline & DBBDF & $1 \mathrm{E} 2$ & 4.3364E-03 & $4.0449 \mathrm{E}-05$ \\
\hline & BBDF2- $\alpha=-0.3$ & $1 \mathrm{E} 2$ & $4.3675 \mathrm{E}-03$ & $5.2938 \mathrm{E}-05$ \\
\hline & BBDF2- $\alpha=0.3$ & $1 \mathrm{E} 2$ & 4.3263E-03 & $3.8130 \mathrm{E}-05$ \\
\hline \multirow[t]{7}{*}{$10^{-4}$} & $\mathrm{BDF}$ & $2 \mathrm{E} 4$ & $2.0965 \mathrm{E}-02$ & $3.2387 \mathrm{E}-04$ \\
\hline & 5-GEAR & $2 \mathrm{E} 4$ & $1.1420 \mathrm{E}-03$ & $7.3623 \mathrm{E}-06$ \\
\hline & $\mathrm{BBDF}$ & $1 \mathrm{E} 4$ & $4.1638 \mathrm{E}-02$ & $6.4178 \mathrm{E}-04$ \\
\hline & 5-DBBDF & $1 \mathrm{E} 4$ & $6.7873 \mathrm{E}-06$ & $1.2180 \mathrm{E}-07$ \\
\hline & DBBDF & $1 \mathrm{E} 4$ & 4.3343E-06 & $7.7818 \mathrm{E}-08$ \\
\hline & BBDF2- $\alpha=-0.3$ & $1 \mathrm{E} 4$ & 4.1057E-06 & $7.3735 \mathrm{E}-08$ \\
\hline & BBDF2- $\alpha=0.3$ & $1 \mathrm{E} 4$ & 4.3481E-06 & $7.4522 \mathrm{E}-08$ \\
\hline \multirow[t]{7}{*}{$10^{-6}$} & $\mathrm{BDF}$ & $2 \mathrm{E} 6$ & $2.1189 \mathrm{E}-04$ & $3.2810 \mathrm{E}-06$ \\
\hline & 5-GEAR & $2 \mathrm{E} 6$ & $1.1902 \mathrm{E}-07$ & $7.6395 \mathrm{E}-10$ \\
\hline & $\mathrm{BBDF}$ & $1 \mathrm{E} 6$ & 4.2374E-04 & $6.5613 \mathrm{E}-06$ \\
\hline & 5-DBBDF & $1 \mathrm{E} 6$ & 7.0587E-10 & $1.4152 \mathrm{E}-11$ \\
\hline & DBBDF & $1 \mathrm{E} 6$ & $6.6448 \mathrm{E}-10$ & $1.9575 \mathrm{E}-11$ \\
\hline & BBDF2- $\alpha=-0.3$ & $1 \mathrm{E} 6$ & $3.8706 \mathrm{E}-10$ & $5.9961 \mathrm{E}-12$ \\
\hline & BBDF2- $\alpha=0.3$ & $1 \mathrm{E} 6$ & $9.8598 \mathrm{E}-10$ & $2.9594 \mathrm{E}-11$ \\
\hline
\end{tabular}

whose exact solution: $y(x)=\frac{8 \sqrt{7}}{175} e^{-\left(\frac{125}{2}\right) x} \sin \left(\frac{25 \sqrt{7}}{2} x\right)$.

Reduction to the system of first order equations:

$$
\begin{gathered}
y_{1}{ }^{\prime}(x)=y_{2}(x), y_{2}{ }^{\prime}(x)=-5000 y_{1}(x)-125 y_{2}(x), \\
y_{1}(0)=0, y_{2}(0)=4,0 \leq x \leq 2,
\end{gathered}
$$

whose exact solutions are

$$
\begin{aligned}
& y_{1}(x)=\frac{8 \sqrt{7}}{175} e^{-\left(\frac{125}{2}\right) x} \sin \left(\frac{25 \sqrt{7}}{2} x\right), \\
& y_{2}(x)=4 e^{-\left(\frac{125}{2}\right) x} \cos \left(\frac{25 \sqrt{7}}{2} x\right)-\frac{20}{\sqrt{7}} e^{-\left(\frac{125}{2}\right) x} \sin \left(\frac{25 \sqrt{7}}{2} x\right) .
\end{aligned}
$$

Table 1. Numerical results for Problem 1

Table 2. Numerical results for Problem 2 
Table 3. Computational time for Problem 1 and Problem 2

\begin{tabular}{cccc}
\hline$h$ & Methods & \multicolumn{2}{c}{ TIME (microseconds) } \\
\hline \multirow{2}{*}{$10^{-2}$} & & Problem 1 & Problem 2 \\
& BDF & 179 & 564 \\
& BBDF & 443 & 314 \\
& BBDF2- $\alpha=-0.3$ & 152 & 498 \\
& BBDF2- $\alpha=0.3$ & 150 & 490 \\
\hline \multirow{2}{*}{$10^{-4}$} & BDF & 13269 & 11973 \\
& BBDF & 13697 & 12191 \\
& BBDF2- $\alpha=-0.3$ & 11215 & 10751 \\
& BBDF2- $\alpha=0.3$ & 10732 & 10283 \\
\hline \multirow{2}{*}{$10^{-6}$} & BDF & 1340928 & 1168904 \\
& BBDF & 1368245 & 1226881 \\
& BBDF2- $\alpha=-0.3$ & 1050992 & 1002043 \\
& BBDF2- $\alpha=0.3$ & 1039905 & 1013512 \\
\hline
\end{tabular}

\section{Discussion}

From Tables $1-2$, it is apparent that the BBDF2- $\alpha$, DBBDF, 5-DBBDF and BBDF take half the total steps as compared to the BDF and 5-GEAR. This is expected because block methods compute the solutions at two points concurrently while the 5-GEAR and BDF are non-block methods, which compute the solutions only at one point for each step. There are no results for BDF and BBDF in Table 1 when $h=10^{-2}$ because the oscillation of the system of ODEs is growing which decreasing the possibility of producing realistic results with these methods. From Table 2 , the BBDF produces very large value of maximum error. This means that this method requires smaller step size to achieve better accuracy at $h=10^{-2}$. Even though the results of maximum error between BBDF- $\alpha$, 5-DBBDF and DBBDF are comparable, the BBDF2- $\alpha$ manages to achieve better accuracy in terms of average error at $h=10^{-6}$. In fact, the derived method still outperforms the BDF, 5-GEAR and DBBDF for both tested problems. The values of MAXE and AVER are depending on the step size. A smaller step size leads to better accuracy, but requires greater computational time. The values of parameters chosen are $\alpha=-0.3,0.3$. Other selections give slightly larger error but for better accuracy must be less than or equal 0.3 .

From Table 3, most of the computational times taken by the method with parameter are observed to be less than the $\mathrm{BDF}$ and BBDF. This is due to its ability in solving the second order problems directly. Meanwhile, BDF and BBDF methods are the first order solvers, which require additional time when solving a system of first order ODEs in the form of double dimensions. It has to be noted that the computation time for the 5-GEAR, DBBDF and 5-DBBDF is not presented due to the difference of computer equipment and software usage.

\section{Conclusions}

The performance of the BBDF2- $\alpha$ presented in this paper on a second order IVPs of ODEs clearly shows its potential for solving second order ODEs directly. Despite the fact that the method is of order 3 , it has been compared with those fifth order methods. From the numerical results obtained, the BBDF2- $\alpha$ has shown competitive accuracy when compared to the higher order existing methods and outstanding computational time over the first order ODEs solvers. Therefore, the direct block method with some appropriate values of independent parameter is preferable for solving second order ODEs.

\section{Acknowledgements}

The publication fee was funded by Geran Penyelidikan Khas UiTM 2020 (600-RMC/GPK 5/3 (272/2020)), Universiti Teknologi MARA Shah Alam. This research was also supported by Universiti Putra Malaysia.

\section{REFERENCES}

[1] C. W. Gear, Numerical initial value problems in ordinary differential equations. Englewood Cliffs, New Jersey.: Prentice-Hall, 1971.

[2] Z. B. Ibrahim, K. I. Othman, and M. Suleiman, "Implicit r-point block backward differentiation formula for solving first-order stiff ODEs," Applied Mathematics and Computation, vol. 186, no. 1, pp. 558-565, 2007, doi: 10.1016/j.amc.2006.07.116.

[3] F. Ismail, Y. L. Ken, and M. Othman, "Explicit and implicit 3-point block methods for solving special second order ordinary differential equations directly," International Journal of Mathematical Analysis, vol. 3, no. 5-8, pp. 239-254, 2009.

[4] F. Oghenerukevwe Obarhua and S. Jacob Kayode, "Continuous explicit hybrid method for solving second order ordinary differential equations," Pure and Applied Mathematics Journal, vol. 9, no. 1, p. 26, 2020, doi: 10.11648/j.pamj.20200901.14.

[5] D. O. Awoyemi, E. A. Adebile, A. O. Adesanya, and T. A. Anake, "Modified order block method for the direct solution second order ordinary differential equations," International Journal of Applied Mathematics and Computation, vol. 3, no. 3, pp. 181-188, 2011.

[6] O. Adeyeye and Z. Omar, "Generalised modified taylor series approach of developing k-step block methods for solving second order ordinary differential equations," Mathematics and Statistics, vol. 8, no. 6, pp. 773-781, 2020, doi: $10.13189 / \mathrm{ms} .2020 .080618$.

[7] M. Suleiman, Z. B. Ibrahim, and A. F. N. Rasedee, "Solution of higher-order ODEs using backward difference method," Mathematical Problems in Engineering, vol. 2011, 2011, doi: $10.1155 / 2011 / 810324$.

[8] M. S. Mechee and N. Senu, "A new numerical multistep method for solution of second order of ordinary differential 
equations," Asian Transactions on Science Technology, vol. 02, no. 02 , pp. 18-22, 2012.

[9] N. Zainuddin, Z. B. Ibrahim, K. I. Othman, and M. Suleiman, "Direct fifth order block backward differentiation formulas for solving second order ordinary differential equations," Chiang Mai Journal of Science, vol. 43, no. 5, pp. 1171-1181, 2016.

[10] E. A. Celaya and J. J. Anza, "BDF- $\alpha$ : A Multistep Method with Numerical Damping Control," Universal Journal of Computational Mathematics, vol. 1, no. 3, pp. 96-108, 2013, doi: 10.13189/ujcmj.2013.010305.

[11] I. S. M. Zawawi and Z. B. Ibrahim, "Derivation of BBDF- $\alpha$ for solving ordinary differential equation," AIP Conference Proceedings, vol. 1750, 2016, doi: 10.1063/1.4954531.

[12] I. S. M. Zawawi and Z. B. Ibrahim, "BBDF- $\alpha$ for solving stiff ordinary differential equationswith oscillating solutions," Tamkang Journal of Mathematics, vol. 51, no. 2, pp. 123-136, 2020, doi: 10.5556/j.tkjm.51.2020.2964.
[13]Z. B. Ibrahim and I. S. M. Zawawi, "A stable fourth order block backward differentiation formulas $(\alpha)$ for solving stiff initial value problems," ASM Science Journal, vol. 12, no. Special Issue 6, pp. 60-66, 2019.

[14] J. Emmanuel and V. Alexander Okhuese, "Derivation and Implementation of a Linear Multistep Numerical Scheme of Order 12," International Journal of Scientific Research in Computer Science, Engineering and Information Technology, no. July, pp. 1117-1127, 2020, doi: 10.32628/cseit2063220.

[15] C. W. Gear, "Numerical integration of ordinary differential equations," Mathematics of Computation, vol. 21, no. 98, pp. 146-156, 1967.

[16]Z. B. Ibrahim, "Block multistep methods for solving ordinary differential equations," Universiti Putra Malaysia, 2006.

[17] N. Zainuddin, "2-point block backward differentiation formula for solving higher order ODEs," Universiti Putra Malaysia, 2011. 